\title{
COMPARISION OF QUANTITATIVE AND QUALITITATIVE ASSESSMENT OF MRI IN DIAGNOSING HIPOCAMPAL ATROPHY AMONG CASES OF TEMPORAL EPILEPSY.
}

1. MBBS, FCPS, FRCP

Fellowship of Intervention Radiology (AKU, Karachi) Senior Registrar

Allied Hospital, Faisalabad.

2. MBBS

Medical Officer

Madinah Teaching Hospital, Faisalabad.

3. MBBS

Medical Officer

Allied Hospital, Faisalabad

4. MBBS

Medical Officer

Allied Hospital, Faisalabad

Correspondence Address: Dr. Muhammad Ahsan Allied Hospital, Faisalabad. ahsanjahangir194@gmail.com

Article received on: 19/11/2018 Accepted for publication: 22/03/2019

Received after proof reading: $25 / 06 / 2019$

\section{Abdul Raouf', Saba Jehangir ${ }^{2}$, Asma Shaukat ${ }^{3}$, Muhammad Ahsan ${ }^{4}$}

ABSTRACT... Mesial temporal sclerosis (MTS) is the most common pathology in patients undergoing anterior temporal lobectomy. Magnetic resonance imaging (MRI) is valuable in detecting MTS. Reduced hippocampal volume and elevated T2 signal are associated with MTS, and both quantitative T2 and volumetric measurements have been associated with hippocampal cellular loss that characterizes this condition. Objectives: To determine the accuracy of hippocampal quantitative (T2 relaxometric) assessment in diagnosing hippocampal atrophy in patients with temporal lobe epilepsy by comparing it with qualitative (visual) assessment on MRI. Study Design: Cross sectional study. Setting: Radiology department of Allied Hospital Faisalabad. Period: 12 months from the approval from Sep, 2016 to Dec, 2017. Subjects \& Methods: After taking permission from hospital ethical committee, and written informed consent, patients with history of temporal lobe epilepsy and EEG findings consistent with temporal lobe epilepsy were examined on 1.5 Tesla Achieva philips scanner, visual assessment and T2 relaxometry. Section of the hippocampus head was defined as the first in which it was possible to see the temporal horn of the lateral ventricle and therefore to appropriately separate the hippocampal formation from the amygdala. The body of the hippocampus defined in the fourth coronal section after the region of interest of the hippocampus head, and the tail was defined in the third coronal section after the hippocampus body, in which it is also possible to visualize the quadrigeminal plate (section of $5 \mathrm{~mm}$ ).Visually the images were assessed and MRI examination was done. All the data was collected on a performa. Results: We concluded that the frequency of accuracy of hippocampal quantitative (t2 relaxometric) assessment in diagnosing hippocampal atrophy in patients with temporal lobe epilepsy by comparing it with qualitative (visual) assessment on MRI is high but needs validation through some-other studies. Conclusion: We concluded that the frequency of accuracy of hippocampal quantitative (t2 relaxometric) assessment in diagnosing hippocampal atrophy in patients with temporal lobe epilepsy by comparing it with qualitative (visual) assessment on MRI is high but needs validation through some-other studies.

Key words: $\quad$ Accuracy, Body, Head, Hippocampus, Hippocampal Quantitative (T2 Relaxometric), Temporal Lobe Epilepsy, Tail.

Article Citation: Raouf A, Jahangir S, Shaukat A, Ahsan M. Comparision of quantitative and qualititative assessment of mri in diagnosing hipocampal atrophy among cases of temporal epilepsy. Professional Med J 2019; 26(7):1009-1013. DOI: 10.29309/TPMJ/2019.26.07.1222

\section{INTRODUCTION}

Epilepsy is one of the chronic diseases of CNS with an enduring predisposition to generate spontaneous epileptic seizures. According to an estimate, about 3 million people in United States are affected by epilepsy and has become one of the most prevalent CNS disorder. Worldwide, an estimated 50 million individuals are affected by epilepsy, which accounts for an overall $1 \%$ burden ${ }^{1}$ with mesial temporal lobe epilepsy
(MTLE) being more prevalent among adults ${ }^{2}$ and in those with intractable temporal lobe epilepsy. ${ }^{3}$

Hippocampal sclerosis (HS) is the main cause of TLE, and up to $65 \%$ of cases of TLE can be attributed to pathology arising entirely in the hippocampus. ${ }^{3}$ Magnetic resonance imaging (MRI) is the ideal investigation for diagnosis of anatomical cause of epilepsy and to look for any associated pathologies and to plan any procedure 
to control refractory cases.

According to the guidelines of United Kingdom, MRI must be done; only exception are children and young adults with generalized epilepsy of no known cause and those showed no improvement after medical therapy (National Institute for Health and Clinical Excellence, 2012). ${ }^{4}$ With the widespread use of high-resolution MRI with epilepsy protocols over the last two decades, our ability to visualize subtle structural changes has been greatly enhanced. ${ }^{5}$ Visual (qualitative) assessment of T2-weighted changes (hyper intense signals and atrophy) is one of the earliest methods which showed association between hippocampal pathology and abnormality identified on MRI. T2 relaxometry is a quantitative technique to determine the prevalence and status of T2 abnormality. ${ }^{3}$ Carr-Purcell-MeiboomGill sequence was used to see the T2 relaxation times. $^{3}$

Comparison of Hippocampal T2 maps from healthy and epileptic individuals shows the prolonged T2 of the HS patients. ${ }^{6}$ Hippocampal sclerosis was detected by visual assessment and T2 relaxometry in $62 \%$ of patients (according to research carried out by Paradeep Singh, Rupinderjeet Kaur, Kavita Saggar, Gagandeep Singh, and Amarpreet Kaur at Bio Med Research International) ${ }^{3}$

This Study is carried out to see how accurately different MRI imaging modalities detect hippocampal sclerosis and quantify disease progression with special benefit to patients who have medically refractory epilepsy and are potential candidates for surgical cure of their illness. Since no similar study has been carried out in Pakistan it is essential that such a study is initiated to benefit patients with both medically treated and medically refractory Epilepsy with better diagnosis and help patient management and prognosis in future.

\section{MATERIAL AND METHODS}

This cross sectional study was conducted after taking permission from hospital ethical committee patients were collected from OPD of Radiology
Department, Allied hospital Faisalabad. Written Informed consent was taken after explaining the objective \& procedure of the study. Patients of age 10-60 years of both sexes having temporal lobe epilepsy on the basis of history and /Or EEG findings were included in the study.

Diagnosed cases of temporal lobe epilepsy confirmed by listening to how they describe their seizures or hearing observations of a witness. Features that were looked for (any one or in combination) are abnormal sensations i.e déjà vu (familiar feeling regarding an incident), jamais vu (unfamiliar feeling or sensation regarding an incident), auditory sensation (an unusual sound or noise), gustatory sensation (an unusual flavor), olfactory (an unusual smell), visual, sensory (presence of something on skin or in the internal organs) and amnesia or resurfacing of old memories and or EEG showing specific 5-7 Hz discharging waves during seizures and ipsilateral anterior /mid temporal inter-ictal spikes or sharp waves suggested the diagnosis. (all above features are for temporal lobe epilepsy).

T2 relaxometry was defined as mapping which provides a quantitative mean for assessment of hippocampal sclerosis. T2 relaxation time of hippocampus ranges from 99 to $106 \mathrm{msec}$ (>116 msec was considered MRI evidence of hippocampal sclerosis). Visual assessment was the assessment of images qualitatively for hippocampal atrophy, loss of defined morphologic structure of hippocampus, the asymmetry between the two hippocampi. and subjective increase in T2 signals and decreased T1 signals. (dark hyper intense as compare to other side). True positives was defined as cases that had hippocampal atrophy both on visual and $\mathrm{T} 2$ relaxometric assessment. True negatives were defined as cases that was not hippocampal atrophy on visual and T2 relaxometric assessment. Patients having history of implanted metallic pacemakers and patients having with claustrophobia were not included in the study.

Sample size of 95 by using the WHO sample size calculator having $\mathrm{P}=62 \%$ and Confidence level of $95 \%$ with absolute precision requirement 
of $10 \%$. Sample collection done through nonprobability consecutive sampling. The patients were examined on Achievaphilips scanner; visual assessment and T2 relaxometry was calculated using 8-echo Carr-Purcell-Meiboom-Gill sequence. Images were taken from the oblique coronal slice from $22 \mathrm{~ms}$ to $352 \mathrm{~ms}$ and along with T2 maps. T2 relaxation time was noted after calculation along with the mean hippocampal T2 relaxation time. Some ROls were marked as hippocampal head, body, and tail; area with temporal horn of lateral ventricle was labeled as head, body was taken in the 4th coronal section and tail in the third coronal section. Visually the images were assessed as per operational definition. The MRI examination was carried out by a senior technician under supervision. The data was collected on a performa.

All the data was analyzed by using SPSS V -16 . Mean and standard deviation was for variables like age and frequency/percentage was calculated for gender and true positive. Effect modifiers like age and gender were controlled through stratification and post-stratification chi-square test applied with p-value of $<0.05$ taken as significant.

\section{RESULTS}

Almost 95 cases meeting criteria were included to determine the accuracy of hippocampal quantitative (T2 relaxometric) assessment in diagnosing hippocampal atrophy in TLE patients by comparing it with qualitative (visual) assessment on MRI. Age distribution and gender is shown in the Table-I and II. Frequency of accuracy of hippocampal quantitative (T2 relaxometric) assessment in diagnosing hippocampal atrophy in patients with TLE by comparing it with qualitative (visual) assessment on MRI was recorded as $72.63 \%(n=69)$ while $27.37 \%(n=26)$ were not diagnosed accurately. (Table-III).

\begin{tabular}{|l|c|c|}
\hline \multicolumn{1}{|c|}{ Age (in Years) } & No. of Patients & $\%$ \\
\hline $10-30$ & 41 & 43.16 \\
\hline $31-60$ & 54 & \multicolumn{2}{c|}{56.84} \\
\hline Total & 95 & 100 \\
\hline Mean+SD & \multicolumn{2}{|c|}{$34.27+9.16$} \\
\hline
\end{tabular}

Table-I. Age distribution $(\mathrm{n}=95)$

\begin{tabular}{|l|c|c|}
\hline \multicolumn{1}{|c|}{ Gender } & No. of Patients & $\%$ \\
\hline Male & 49 & 51.58 \\
\hline Female & 46 & \\
\hline Total & 95 & 100 \\
\hline
\end{tabular}

Table-II. Gender distribution $(n=95)$

\begin{tabular}{|l|c|c|}
\hline \multicolumn{1}{|c|}{ Accuracy } & No. of Patients & $\%$ \\
\hline Yes & 69 & 72.63 \\
\hline No & 26 & 27.37 \\
\hline Total & 95 & 100 \\
\hline
\end{tabular}

Table-III. Frequency of accuracy of hippocampal quantitative (T2 relaxometric) assessment in diagnosing hippocampal atrophy in patients

with temporal lobe epilepsy by comparing it with qualitative (visual) assessment on MRI $(n=95)$

Effect modifiers like age and gender were calculated by stratification. Post stratification chisquare test was applied. P-value $<0.05$ was taken as significant. (Table-IV\&V).

\begin{tabular}{|c|c|c|c|}
\hline \multirow{2}{*}{$\begin{array}{c}\text { Age } \\
\text { (in Years) }\end{array}$} & \multicolumn{2}{|c|}{$\begin{array}{c}\text { Hippocampal atrophy in } \\
\text { patients with temporal } \\
\text { lobe epilepsy }\end{array}$} & P-Value \\
\cline { 2 - 3 } & Yes & No & \\
\hline $10-30$ & 32 & 9 & 0.30 \\
\hline $31-60$ & 37 & 17 & 0.30 \\
\hline
\end{tabular}

Table-IV. Stratification for frequency of accuracy with regards to age $(n=95)$

\begin{tabular}{|l|c|c|c|}
\hline $\begin{array}{c}\text { Age } \\
\text { (in Years) }\end{array}$ & \multicolumn{2}{|c|}{$\begin{array}{c}\text { Hippocampal atrophy in } \\
\text { patients with temporal } \\
\text { lobe epilepsy }\end{array}$} & \multirow{2}{*}{ P-Value } \\
\cline { 1 - 2 } & Yes & No & \\
\hline Male & 35 & 14 & \multirow{2}{*}{0.78} \\
\hline Female & 34 & 12 & \\
\hline Table-V. Stratification for frequency of accuracy with \\
regards to gender (n=95)
\end{tabular}

\section{DISCUSSION}

Patients with mesial temporal sclerosis (MTS) end up in a procedure called anterior temporal lobectomy. MRI is ideal for the detection of MTS. MRI showing decrease in size of hippocampus and increased T2 signal is significantly associated with MTS; both these are further due to loss of cells in the hippocampus.

This Study was carried out to see how accurately different MRI imaging modalities detect 
hippocampal sclerosis and quantify disease progression with special benefit to patients who have medically refractory epilepsy and are potential candidates for surgical cure of their illness. Since no similar study has been carried out in Pakistan it is essential that such a study is initiated to benefit patients with both medically treated and medically refractory Epilepsy with better diagnosis and help patient management and prognosis in future.

In this study, out of 95 cases, $43.16 \%(n=41)$ were between $10-30$ years of age while $56.84 \%(n=54)$ were between 31-60 years of age, mean + SD was calculated as $34.27+9.16$ years, $51.58 \%$ $(n=49)$ were male and $48.42 \%(n=46)$ were females. Frequency of accuracy of hippocampal quantitative (T2relaxometric) assessment in diagnosing hippocampal atrophy in TLE cases by comparing it with qualitative (visual) assessment on MRI was recorded as $72.63 \%(n=69)$.

SinghP, and others are of the view that hippocampal sclerosis was detected by visual assessment and T2 relaxometry in $62 \%$ of patients. ${ }^{3}$ Wehner et $\mathrm{al}^{7}$ investigated patients with MTLE and concluded that the apparent diffusion coefficients did not provide any lateralizing information without any evidence of hippocampal sclerosis at MR imaging performed with a standard temporal lobe protocol.

Capizzano et $\mathrm{al}^{8}$ reported that, by using only hippocampal data with proton MR spectroscopy, $60 \%$ of cases of MTLE were correctly lateralized; this MR spectroscopy result is lower than the sensitivity of the finding of PLHS. Jackson et al. ${ }^{9}$ concluded that about $15 \%$ cases with hippocampal sclerosis showed hippocampus volumes within normal range on MRI.

Cohen-Gadol et $\mathrm{al}^{10}$ designated the group of patients whose features and labs favor MTLE and MR imaging had no signal intensity abnormality at hippocampus as having paradoxical temporal lobe epilepsy (PTLE). In their evaluation, loss of pyramidal cells in the CA1 region was significantly slighter for PTLE than for typical hippocampal sclerosis. The cases showing only PLHS as an abnormal finding in our study might coincide with the cases of PTLE described by Cohen-Gadol et al. Since no similar study has been carried out in Pakistan it is essential to conduct some other studies to validate our results.

\section{CONCLUSION}

We concluded that the frequency of accuracy of hippocampal quantitative (T2 relaxometric) assessment in diagnosing hippocampal atrophy in cases with TLP by comparing it with qualitative (visual) assessment on MRI is high but needs validation through some-other studies

\section{CONFLICT OF INTEREST}

None of the authors have declared any conflict of interest.

\section{Copyright $\odot 22$ Mar, 2019.}

\section{REFERENCES}

1. Memarian N, Thompson PM, Engel Jr J, Staba RJ. Quantitative analysis of structural neuroimaging of mesial temporal lobe epilepsy. Imaging in medicine. 2013 Jun 1;5(3).

2. Coan AC1, Kubota B, Bergo FP, Campos BM, Cendes F. 3T MRI quantification of hippocampal volume and signal in mesial temporal lobe epilepsy improves detection of hippocampal sclerosis. AJNR AM J Neuroradiol 2014; 35:77-83.

3. Singh P, Kaur R, Saggar K, Sing G, Kaur A. Qualitative and quantativehippocampalMRlassessmentin intractable epilepsy. Biomed Resnlnt. 2013; 480:524.

4. Winston GP, Micallef C, Kendell BE, Bartlett PA, Williams $E J$, Burdett JL, et al. The value of repeat neuroimaging for epilepsy at tertiary refferal center: 16 years of experience. Epilepsy Res. 2013; 105:349-55.

5. Shah AK, Mittal S. Evaluation of magnetic resonance imaging-negative drug-resistant epilepsy. Ann Indian Acad Neurol. 2014; 17:80-8.

6. Deoni SCL. Quantitative relaxometry of the brain. Top Magn Reson Imaging. 2010; 21:101-13.

7. Wehner T, Lapresto $E$, Tkach J, et al. The value of interictal diffusion-weighted imaging in lateralizing temporal lobe epilepsy. Neurology 2007; 68:122-7.

8. Capizzano AA, Vermathen P, Laxer KD. Multisection proton MR spectroscopy for mesial temporal lobe epilepsy. AJNR Am J Neuroradiol 2002; 23:1359-68. 
9. Jackson GD, Kuzniecky RI, Cascino GD. Hippocampal sclerosis without detectable hippocampal atrophy. Neurology 1994; 44: 42-6.
10. Cohen-Gadol AA, Bradley CC, Williamson A. Normal magnetic resonance imaging and medial temporal lobe epilepsy: the clinical syndrome of paradoxical temporal lobe epilepsy. J Neurosurg 2005; 102:902-9.

\begin{tabular}{|c|l|l|}
\hline \multicolumn{3}{|c|}{ AUTHORSHIP AND CONTRIBUTION DECLARATION } \\
\hline Sr. \# & \multicolumn{1}{|c|}{ Author-s Full Name } & \multicolumn{1}{|c|}{ Contribution to the paper } \\
\hline 1 & Abdul Raouf & Data collection. \\
\hline 2 & Saba Jehangir & Data collection. \\
\hline 3 & Asma Shaukat & Data analysis \& Data collection. \\
\hline 4 & Muhammad Ahsan & Paper writing \& Data analysis. \\
\hline
\end{tabular}

